\title{
On the partition function of the six-vertex model with domain wall boundary conditions
}

\author{
F. Colomo \\ I.N.F.N., Sezione di Firenze \\ and Dipartimento di Fisica, Università di Firenze \\ Via G. Sansone 1, 50019 Sesto Fiorentino (FI), Italy
}

A.G. Pronko

Steklov Institute of Mathematics at St Petersburg, Fontanka 27, 191023 St Petersburg, Russia

\begin{abstract}
The six-vertex model on an $N \times N$ square lattice with domain wall boundary conditions is considered. A Fredholm determinant representation for the partition function of the model is given. The kernel of the corresponding integral operator is of the so-called integrable type, and involves classical orthogonal polynomials. From this representation, a "reconstruction" formula is proposed, which expresses the partition function as the trace of a suitably chosen quantum operator, in the spirit of corner transfer matrix and vertex operator approaches to integrable spin models.
\end{abstract}




\section{Introduction}

The six-vertex model on a square lattice with domain wall boundary conditions (DWBC) was introduced in [1] and subsequently solved in [2], where a determinant formula for the partition function was obtained and proven (see also [3]). This model, in its inhomogeneous formulation (i.e. with the vertex weights given as suitable functions of the position of the vertex on the lattice), naturally arises when investigating correlation functions of quantum integrable models in the framework of quantum inverse scattering method. In its homogeneous version, the model admits usual interpretation as a model of statistical mechanics with fixed boundary conditions, and may be seen as a variation of the original sixvertex model with periodic boundary conditions [4-7], which has been for decades a paradigmatic one in statistical mechanics [8].

The DWBC version of the six-vertex model has however several non trivial peculiarities which render it worthy of further investigations. Firstly, the model with this very specific fixed boundary conditions enjoys interesting connections with some important issues of enumerative combinatorics, such as Alternating Sign Matrices [9-11] and domino tilings [12,13]. Moreover, it appears that, even in the thermodynamic limit, bulk quantities, such as the bulk free energy, are indeed sensitive to the choice of boundary conditions $[14,15]$ (see also [16]). Finally, the determinant representation given in [2,3] for the partition function, and analogous ones recently presented for the boundary one-point correlation functions (polarizations) [17], are rather implicit and turn out to be too intricate for any further, more explicit, answer, except in very particular cases. Alternative equivalent representations for the partition function and polarizations would be therefore highly desirable to address several problems, such as further Alternating Sign Matrices weighted enumerations, or extensions of the Arctic Circle Theorem [13] beyond the Free-Fermion point.

The model is formulated on a square lattice with arrows on edges. The only admitted configurations are such that there are always two arrows pointing away from, and two arrows pointing into, each lattice vertex; each vertex can therefore be in one out of six different possible states, a Boltzmann weight $w_{i}$ being assigned to each vertex, according to its state $i(i=1, \ldots, 6)$. We shall consider here the homogeneous version of the model, where the Boltzmann weights are site independent. The DWBC are imposed on the $N \times N$ square lattice by fixing the direction of all arrows on the boundaries as follow: the vertical arrows on the top and bottom of the lattice point inward, while the horizontal arrows on the 

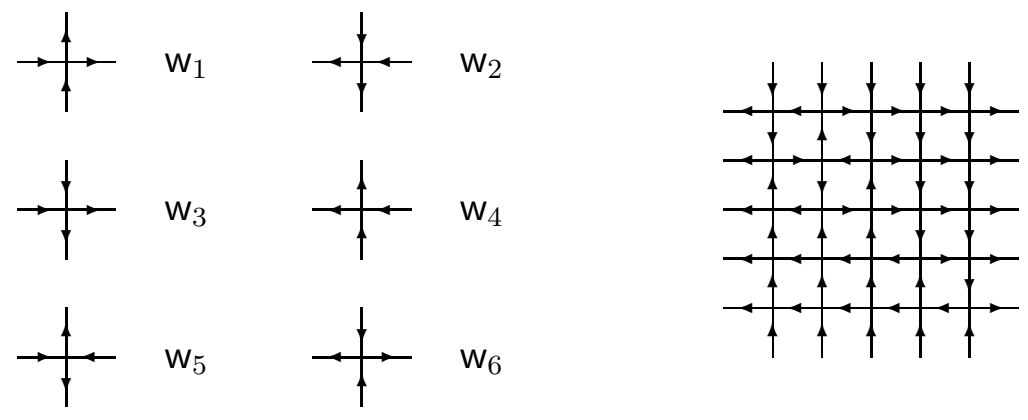

Figure 1: The six allowed types of vertices, their weights and one of the possible configurations in the model with the domain wall boundary conditions for $N=5$.

left and right sides point outward. The correspondence between the Boltzmann vertex weights and the arrow configurations, and a typical configuration of the model with DWBC, are shown in Fig. 1.

The partition function is obtained by summing over all possible arrow configurations, compatible with the imposed DWBC, each configuration being assigned its Boltzmann weight, given simply as the product of all the corresponding vertex weights:

$$
Z_{N}=\sum_{\text {DWBC configurations }} \prod_{i=1}^{6} \mathrm{w}_{i}^{n_{i}} .
$$

Here $n_{i}$ denotes the number of vertices in state $i$, i.e. with Boltzmann weight $\mathrm{w}_{i}$, in each configuration, and $\sum_{i=1}^{6} n_{i}=N^{2}$. The six-vertex model with DWBC is usually considered with its weights invariant under inversion of all arrows, and thus with only three distinct weight functions, denoted as a, b and c,

$$
\mathrm{w}_{1}=\mathrm{w}_{2} \equiv \mathrm{a}, \quad \mathrm{w}_{3}=\mathrm{w}_{4} \equiv \mathrm{b}, \quad \mathrm{w}_{5}=\mathrm{w}_{6} \equiv \mathrm{c}
$$

We shall use the following parametrization for the weight functions

$$
\mathrm{a}=\sin (\lambda+\eta), \quad \mathrm{b}=\sin (\lambda-\eta), \quad \mathrm{c}=\sin 2 \eta
$$

In terms of this parametrization the result of Ref. [3] for the partition function reads

$$
Z_{N}=\frac{[\sin (\lambda-\eta) \sin (\lambda+\eta)]^{N^{2}}}{\prod_{k=1}^{N-1}(k !)^{2}} \operatorname{det}_{N} H
$$


where $H$ is an $N \times N$ Hänkel matrix, with entries

$$
H_{j k}=\frac{\partial^{j+k}}{\partial \lambda^{j+k}} \frac{\sin 2 \eta}{\sin (\lambda-\eta) \sin (\lambda+\eta)} .
$$

Here and in the following we use the convention that indices of $N \times N$ matrices run over the values $j, k=0,1, \ldots, N-1$. Formula (1.4) for the partition function, which was originally obtained within the Quantum Inverse Scattering Method, will be referred to as the Hänkel determinant representation.

The purpose of the present paper is to give some other equivalent representations for the partition function. The emphasis will be made on the representation in terms of Fredholm determinant of some linear integral operator of integrable type, in the sense of paper [18]. Representations of such type have been proven to be powerful tools in many areas of mathematical physics, ranging from the theory of Random Matrices to the asymptotics of Orthogonal Polynomials. Among them is the theory of correlation functions of quantum integrable models [19], the area of origin of the six-vertex model with DWBC itself.

The general procedure which we shall follow to build Fredholm determinant formula for the partition function has been suggested in [20]; in contrast to that paper, we shall however apply this procedure directly to the Hänkel determinant representation (1.4). In this way, the Hänkel structure is preserved, and the integrability of the integral operator in the Fredholm determinant is ensured by construction. We therefore propose a simple factorization for the determinant of the Hänkel matrix $H$ appearing in (1.4). This factorization, quite natural in the construction of a Fredholm determinant representation for the partition function, allows moreover to identify a core term in the factorized form of (1.4), with all others factor being trivial, and disappearing with a mere redefinition of vertex weights. The core term turns out to correspond exactly to the partition function for the six-vertex model when its $R$-matrix is specialized to the $U_{q}\left(\mathfrak{s l}_{2}\right)$ invariant $R$-matrix [21], i.e. when its vertex weights are chosen accordingly with its underlying quantum group symmetry. This core term, which we shall denote as $\tilde{Z}_{N}$ gives rise to the Fredholm determinant, thanks to standard techniques from the theory of Orthogonal Polynomials.

Together with this Fredholm determinant representation, we readily get for $\tilde{Z}_{N}$ an equivalent representation as the ordinary determinant of an $N \times N$ symmetric matrix, whose entries can be explicitly evaluated. From this last representation we propose a "reconstruction" formula which expresses the partition function as a trace of some quantum operator, which, in the large $N$ limit, turns into the exponential of the boost operator for free fermions on a lattice. This 
recalls analogous formulae which appears in the framework of corner transfer matrix and vertex operator approaches to integrable lattice models [8,22]. For $N$ finite, a corresponding "reconstruction" formula for $\tilde{Z}_{N}$ can also be written, as the trace (over the Fock space of $N$ canonical fermions) of a product of exponentials of local operators.

\section{The factorization of the Hänkel determinant}

In our way to build a Fredholm representation for the partition function $Z_{N}$ we shall follow the procedure suggested in [20], but applying it directly to the Hänkel determinant representation (1.4). As a consequence, the Hänkel structure is preserved, and the integrability of the integral operator in the Fredholm determinant is ensured by construction. In this section we shall discuss a specific factorization (a somewhat trivial one, but nevertheless basic for what follows) for the determinant of the Hänkel matrix $H$ appearing in (1.4).

All the different equivalent representations for the partition function derived

in this paper stem essentially from the following simple observation: using the identity

$$
\frac{\sin 2 \eta}{\sin (\lambda-\eta) \sin (\lambda+\eta)}=\cot (\lambda-\eta)-\cot (\lambda+\eta)
$$

the matrix $H$ can be naturally written as a difference of two matrices

$$
H=A_{-}-A_{+}, \quad A_{ \pm}=\left.A\right|_{\phi=\phi_{ \pm}}, \quad \phi_{ \pm}=\lambda \pm \eta
$$

where the matrix $A$ can be chosen to be

$$
A_{j k}=\frac{\partial^{j+k}}{\partial \phi^{j+k}}[\cot \phi-\mathrm{i}] \text {. }
$$

Our choice of the additive constant $-\mathrm{i}$ will be explained below; its role is to ensure invertibility of matrix (2.3) for any complex value of $\phi$. The structure of (2.2) suggests to factorize the determinant of $H$, for instance, as follows

$$
\operatorname{det}_{N} H=\operatorname{det}_{N}\left(A_{-}\right) \operatorname{det}_{N}\left(I-A_{-}^{-1} A_{+}\right) .
$$

The most evident consequence of such factorization is of course the relatively natural and straightforward emergence of a Fredholm determinant representation for the partition function, the Fredholm determinant being related to the second factor in (2.4). The proposed factorization however suggests more: as we shall 
discuss in detail in the following (see Sect. 4), a "reconstruction" formula can be deduced, which allows to represent the partition function as the trace of a suitably chosen quantum operator, in the spirit of corner transfer matrix and vertex operator approaches to integrable spin models.

The analysis of factorization (2.4) relies essentially on the properties of matrix $A$ and in particular of its determinant. To this purpose standard techniques relating Hänkel matrices with orthogonal polynomials [23] will be exploited. Let us assume that entries of some generic $N \times N$ Hänkel matrix $A$ are given as

$$
A_{j k}:=\int_{-\infty}^{\infty} x^{j+k} \omega(x) \mathrm{d} x
$$

Let us moreover suppose that there exist a (complete) set of polynomials $p_{n}(x)$, orthonormal with respect to the measure $\omega(x) \mathrm{d} x$ :

$$
\int_{-\infty}^{\infty} p_{j}(x) p_{k}(x) \omega(x) \mathrm{d} x=\delta_{j k}
$$

Then, calling $\kappa_{n}$ the leading coefficient of $p_{n}(x)$,

$$
p_{n}(x)=\kappa_{n} x^{n}+\ldots, \quad \kappa_{n} \neq 0,
$$

the determinant of matrix (2.5) is simply given as

$$
\operatorname{det}_{N} A=\prod_{j=0}^{N-1} \kappa_{j}^{-2} .
$$

Of course, this formula turns out useful provided that the set of orthogonal polynomials associated to the measure $\omega(x) \mathrm{d} x$ can be identified. In the case of matrix $H$ given by (1.5) appropriate polynomials are not available and the previous scheme cannot be fulfilled for generic values of vertex weights. However, the matrix defined in equation (2.3) is much simpler and the scheme can be fulfilled explicitly.

The entries of matrix (2.3) being periodic in $\operatorname{Re} \phi$, we may restrict ourselves to consider values of $\phi$ varying over the vertical strip $0 \leqslant \operatorname{Re} \phi<\pi$ (with the point $\phi=0$ excluded). In this region we may use

$$
\cot \phi=\mathrm{v} \cdot \mathrm{p} \cdot \int_{-\infty}^{\infty} \frac{\mathrm{e}^{\phi x}}{1-\mathrm{e}^{\pi x}} \mathrm{~d} x
$$

to write the entries of matrix $A$, Eq. (2.3), in the form (2.5) with

$$
\omega(x)=\frac{\mathrm{e}^{\phi x}}{1-\mathrm{e}^{\pi x}+\mathrm{i} 0} .
$$


The polynomials $p_{n}(x)$ (depending on $\phi$ as a parameter), associated to the matrix $A$, should therefore satisfy

$$
\int_{-\infty}^{\infty} p_{j}(x) p_{k}(x) \frac{\mathrm{e}^{\phi x}}{1-\mathrm{e}^{\pi x}+\mathrm{i} 0} \mathrm{~d} x=\delta_{j k}
$$

To identify the explicit form of these polynomials we shall now reexpress the orthogonality condition in such a way that the integration contour, though still being the real axis, has no singularity in its vicinity. This can be achieved by shifting the integration contour $\mathbb{R} \rightarrow \mathbb{R}-\mathrm{i}$ and simultaneously relabelling the integration variable: $x \rightarrow x-\mathrm{i}$. The orthogonality condition now reads

$$
\mathrm{e}^{-\mathrm{i} \phi} \int_{-\infty}^{\infty} p_{j}(x-\mathrm{i}) p_{k}(x-\mathrm{i}) \frac{\mathrm{e}^{\phi x}}{1+\mathrm{e}^{\pi x}} \mathrm{~d} x=\delta_{j k} .
$$

Rewriting the weight function as

$$
\frac{\mathrm{e}^{\phi x}}{1+\mathrm{e}^{\pi x}}=\frac{1}{2 \pi} \Gamma\left(\frac{1-\mathrm{i} x}{2}\right) \Gamma\left(\frac{1+\mathrm{i} x}{2}\right) \mathrm{e}^{(\phi-\pi / 2) x}
$$

and comparing (2.12) with the orthogonality condition of Meixner-Pollaczek polynomials [24]

$$
\begin{aligned}
& \frac{1}{2 \pi} \int_{-\infty}^{\infty} P_{n}^{(\lambda)}(x ; \phi) P_{m}^{(\lambda)}(x ; \phi) \Gamma(\lambda-\mathrm{i} x) \Gamma(\lambda+\mathrm{i} x) \mathrm{e}^{(2 \phi-\pi) x} \mathrm{~d} x \\
& \quad=\frac{\Gamma(n+2 \lambda)}{n !(2 \sin \phi)^{2 \lambda}} \delta_{n m}
\end{aligned}
$$

where

$$
P_{n}^{(\lambda)}(x ; \phi)=\frac{(2 \lambda)_{n}}{n !} \mathrm{e}_{2}^{\mathrm{i} n \phi} F_{1}\left(\begin{array}{c}
-n, \lambda+\mathrm{i} x \\
2 \lambda
\end{array} \mid 1-\mathrm{e}^{-2 \mathrm{i} \phi}\right)
$$

we readily identify the polynomials in question with nothing but the MeixnerPollaczek polynomials, where the parameter $\lambda$ (not to be confused with the variable $\lambda$ entering the parametrization of the vertex weights) must be specialized to the value $\lambda=1 / 2$.

Therefore, the polynomials $p_{n}(x)$ satisfying (2.11) are

$$
p_{n}(x)=\mathrm{e}^{\mathrm{i} \phi / 2} \sqrt{\sin \phi} P_{n}^{(1 / 2)}\left(\frac{x+\mathrm{i}}{2} ; \phi\right)
$$

or, due to (2.15), more explicitly

$$
p_{n}(x)=\mathrm{e}^{\mathrm{i}(n+1 / 2) \phi} \sqrt{\sin \phi}{ }_{2} F_{1}\left(\begin{array}{c|c}
-n, \mathrm{i} x / 2 & 1-\mathrm{e}^{-2 \mathrm{i} \phi} \\
1 &
\end{array}\right) .
$$


From this expression the highest coefficient $\kappa_{n}$ of the polynomial $p_{n}(x)$ can be explicitly evaluated

$$
\kappa_{n}(\phi)=\frac{\mathrm{e}^{\mathrm{i} \phi / 2}(\sin \phi)^{n+1 / 2}}{n !},
$$

and, due to formula (2.8), we readily get for the determinant of the matrix $A$ :

$$
\operatorname{det}_{N} A=\frac{\mathrm{e}^{-\mathrm{i} N \phi}}{(\sin \phi)^{N^{2}}} \prod_{n=1}^{N-1}(n !)^{2} .
$$

This last expression shows that if matrix $A$ exists (that is if all its entries are finite) then it is invertible, since its determinant never vanishes (for finite $\phi$ ). Factorization (2.4) therefore leads to an equivalent representation for the partition function, valid for all (non vanishing) values of $\phi_{ \pm}$.

Let us note that our choice of the constant $(-\mathrm{i})$ in the definition of matrix (2.3) can be easily explained now. Indeed, by considering a combination of (2.19) and its formal complex conjugate $(\mathrm{i} \rightarrow-\mathrm{i})$ one finds

$$
\operatorname{det}\left[\frac{\partial^{j+k}}{\partial \phi^{j+k}}(\cot \phi+\alpha)\right]_{j, k=0}^{N-1}=\frac{\cos (N \phi)+\alpha \sin (N \phi)}{(\sin \phi)^{N^{2}}} \prod_{n=1}^{N-1}(n !)^{2} .
$$

The expression $\cos (N \phi)+\alpha \sin (N \phi)$ possesses zeroes in the complex plane of the variable $\phi$ unless $\alpha= \pm \mathrm{i}$. Thus, by choosing $\alpha=-\mathrm{i}$ (or, equivalently, $\alpha=\mathrm{i}$ ) the invertibility of matrix $A$ is ensured.

With (2.19) taken into account the partition function now reads

$$
Z_{N}=\left[\sin \phi_{+}\right]^{N^{2}} \mathrm{e}^{-\mathrm{i} N \phi_{-}} \operatorname{det}_{N}\left(I-A_{-}^{-1} A_{+}\right) .
$$

It is worth emphasizing that the partition function is, in fact, described only by the last factor, the first two factors having a trivial meaning, and disappearing with a mere redefinition of the vertex weights. Indeed, the first factor in (2.21) can be seen as the result of a common prefactor in all weights, and we get rid of it by changing the overall normalization of the weights. The second one is a "boundary" factor specific of the DWBC choice for the six-vertex model; it can be removed by introducing a suitable asymmetry in weights $w_{5}$ and $w_{6}$, since any configuration contributing to the partition function (that is, satisfying both the "ice rule" and the DWBC), is such that the numbers of vertices of these two types satisfy the condition $\# \mathrm{w}_{6}-\# \mathrm{w}_{5}=N$, Hence, by choosing the weights to be

$$
\begin{aligned}
& \mathrm{w}_{1}=\mathrm{w}_{2}=1, \quad \mathrm{w}_{3}=\mathrm{w}_{4}=\mathrm{b} / \mathrm{a}, \\
& \mathrm{w}_{5}=\mathrm{e}^{-\mathrm{i}(\lambda-\eta)} \mathrm{c} / \mathrm{a}, \quad \mathrm{w}_{6}=\mathrm{e}^{\mathrm{i}(\lambda-\eta)} \mathrm{c} / \mathrm{a},
\end{aligned}
$$


with a, b, and c given by (1.3), the partition function reduces to the sole determinant in (2.4).

The choice of the weights (2.22) has a simple interpretation in terms of the six-vertex model $R$-matrix, the matrix of local vertex states. In the standard notations the choice of the weights in the form (2.22) corresponds to the following $R$-matrix

$$
R(\nu)=\left(\begin{array}{cccc}
1 & & & \\
& \beta(\nu) & \mathrm{e}^{\mathrm{i} \nu} \gamma(\nu) & \\
& \mathrm{e}^{-\mathrm{i} \nu} \gamma(\nu) & \beta(\nu) & \\
& & & 1
\end{array}\right)
$$

where

$$
\beta(\nu)=\frac{\sin \nu}{\sin (\nu+2 \eta)}, \quad \gamma(\nu)=\frac{\sin 2 \eta}{\sin (\nu+2 \eta)}, \quad \nu=\lambda-\eta .
$$

In this expression one easily recognizes the $U_{q}\left(\mathfrak{s l}_{2}\right)$-invariant $R$-matrix [21] which is, moreover, normalized to satisfy the "unitarity condition":

$$
R(\nu)(P R(-\nu) P)=I
$$

with the permutation operator $P:=R(0)$. Thus, the factorization (2.4) of the initial Hänkel determinant leads naturally to the representation of the partition function just as the sole determinant in (2.21), provided that the vertex weights are chosen according to the underlying quantum group symmetry of the model. From now on we shall assume this choice, equation (2.22), for the vertex weights, denoting the corresponding partition function as $\tilde{Z}_{N}$, given as

$$
\tilde{Z}_{N}=\operatorname{det}_{N}\left(I-A_{-}^{-1} A_{+}\right)
$$

It is worth mentioning here that almost everything above can also be extended to the case of the determinant formula of Ref. [2] for the partition function of the inhomogeneous model. Without giving any detail, let us just emphasize that previous considerations become even more transparent, since in that (more general) case matrix $A$ is a Cauchy matrix. However, the homogeneous model possess the further interesting property that the partition function can be expressed as the Fredholm determinant of an integral operator of integrable type, in the sense paper of [18]. This will be the subject of the next Section. 


\section{The partition function as a Fredholm determinant}

We shall now focus our attention on the determinant in (2.26). First of all we need to build the entries of matrix $A_{-}^{-1}=\left.A^{-1}\right|_{\phi=\phi_{-}}$. This task can be achieved straightforwardly by borrowing standard techniques from the theory of orthogonal polynomials $[20,23]$. Let us recall that, indeed, once the set of orthogonal polynomials associated to a given Hänkel matrix $A$ with entries (2.5) is known, the entries of the corresponding inverse matrix $A^{-1}$ can be evaluated from the function

$$
\mathcal{K}_{N}(x, y)=\sum_{n=0}^{N-1} p_{n}(x) p_{n}(y) .
$$

simply in terms of partial derivatives:

$$
A_{j k}^{-1}=\left.\frac{1}{j !} \frac{\partial^{j}}{\partial x^{j}} \frac{1}{k !} \frac{\partial^{k}}{\partial y^{k}} \mathcal{K}_{N}(x, y)\right|_{x=0, y=0} .
$$

The proof is based on the fact that function $\mathcal{K}_{N}(x, y)$ is the kernel of an integral operator, with respect to the measure $\omega(x) \mathrm{d} x$. This operator, by construction, projects over the subspace of polynomials of order less than $N$, and therefore acts as identity operator on monomials $1, x, \ldots, x^{N-1}$ :

$$
\int_{-\infty}^{\infty} \mathcal{K}_{N}(x, y) y^{m} \omega(x) \mathrm{d} x=x^{m}, \quad m=0,1, \ldots, N-1 .
$$

With (3.3) taken into account, equation (3.2) can be verified directly:

$$
\begin{aligned}
\sum_{k=0}^{N-1} A_{j k}^{-1} A_{k m} & =\left.\sum_{k=0}^{N-1} \frac{1}{j !} \frac{\partial^{j}}{\partial x^{j}} \frac{1}{k !} \frac{\partial^{k}}{\partial y^{k}} \mathcal{K}_{N}(x, y) \int_{-\infty}^{\infty} z^{k+m} \omega(z) \mathrm{d} z\right|_{x=0, y=0} \\
& =\left.\frac{1}{j !} \frac{\partial^{j}}{\partial x^{j}} \int_{-\infty}^{\infty} \mathcal{K}_{N}(x, z) z^{m} \omega(z) \mathrm{d} z\right|_{x=0} \\
& =\left.\frac{1}{j !} \frac{\partial^{j}}{\partial x^{j}} x^{m}\right|_{x=0} \\
& =\delta_{j m} .
\end{aligned}
$$

In the case of matrix $A(2.3)$ the entries of the inverse matrix $A^{-1}$ are given by (3.2) with the function $\mathcal{K}_{N}(x, y)$ build from formula (3.1) in terms of orthogonal polynomials $p_{n}(x)$, defined in Eq. (2.17).

Generalizing the simple computation above one can evaluate the entries of the product matrix $A_{-}^{-1} A_{+}$:

$$
\left[A_{-}^{-1} A_{+}\right]_{j m}=\left.\frac{1}{j !} \frac{\partial^{j}}{\partial x^{j}} \int_{-\infty}^{\infty} \mathcal{K}_{N}^{-}(x, z) z^{m} \omega^{+}(z) \mathrm{d} z\right|_{x=0} .
$$


Here the + or - superscripts denote the dependence on the variables $\phi_{ \pm}$respectively. The determinant in (2.26) can now be transformed as follows

$$
\begin{aligned}
& \ln \operatorname{det}_{N}\left(I-A_{-}^{-1} A_{+}\right) \\
& \quad=-\sum_{n=1}^{\infty} \frac{1}{n} \operatorname{tr}_{N}\left(A_{-}^{-1} A_{+}\right)^{n} \\
& \quad=-\sum_{n=1}^{\infty} \frac{1}{n} \int_{-\infty}^{\infty} \ldots \int_{-\infty}^{\infty} \mathcal{K}_{N}^{-}\left(x_{1}, x_{2}\right) \mathcal{K}_{N}^{-}\left(x_{2}, x_{3}\right) \ldots \mathcal{K}_{N}^{-}\left(x_{n}, x_{1}\right) \prod_{l=1}^{n} \omega^{+}\left(x_{l}\right) \mathrm{d} x_{l} \\
& =:-\sum_{n=1}^{\infty} \frac{1}{n} \operatorname{tr}\left(\mathcal{V}_{N}\right)^{n} \\
& =\ln \operatorname{det}\left(\mathcal{I}-\mathcal{V}_{N}\right)
\end{aligned}
$$

where $\mathcal{V}_{N}$ is the integral operator on the real axis with kernel

$$
\mathcal{V}_{N}(x, y)=\mathcal{K}_{N}^{-}(x, y) \omega^{+}(y)
$$

Using the Christoffel-Darboux identity, this kernel can be written as

$$
\mathcal{V}_{N}(x, y)=\frac{\kappa_{N-1}^{-}}{\kappa_{N}^{-}} \frac{p_{N}^{-}(x) p_{N-1}^{-}(y)-p_{N-1}^{-}(x) p_{N}^{-}(y)}{x-y} \omega^{+}(y)
$$

rendering the integrability (in the sense of Ref. [18]) of integral operator $\mathcal{V}_{N}$ manifest. Integral kernels of the form (3.8) are also known under the name of correlation kernels since they arise in expressions for eigenvalues correlation functions in the theory of random matrices. For discussion of the role and importance of this special class of integral operators in connection with correlation functions of integrable models and with the theory of random matrix, see [19,25].

Taking into account formulae (2.10), (2.16), (2.17) and (2.18) we therefore get for the partition function of the model, with the choice (2.22) for its vertex weights, the following Fredholm determinant representation:

$$
\tilde{Z}_{N}=\operatorname{det}\left(\mathcal{I}-\mathcal{V}_{N}\right)
$$

where the kernel may be most explicitely written as

$$
\begin{aligned}
\mathcal{V}_{N}(x, y)= & \left\{{ }_{2} F_{1}\left(\begin{array}{c}
-N, \mathrm{i} x / 2 \\
1
\end{array} \mid 1-\mathrm{e}^{-2 \mathrm{i} \phi_{-}}\right){ }_{2} F_{1}\left(\begin{array}{c}
-N+1, \mathrm{i} y / 2 \\
1
\end{array} \mid 1-\mathrm{e}^{-2 \mathrm{i} \phi_{-}}\right)\right. \\
& \left.-{ }_{2} F_{1}\left(\begin{array}{c}
-N+1, \mathrm{i} x / 2 \\
1
\end{array} \mid 1-\mathrm{e}^{-2 \mathrm{i} \phi_{-}}\right){ }_{2} F_{1}\left(\begin{array}{c}
-N, \mathrm{i} y / 2 \\
1
\end{array} \mid 1-\mathrm{e}^{-2 \mathrm{i} \phi_{-}}\right)\right\} \\
& \times \frac{N \mathrm{e}^{2 \mathrm{iN} \phi_{-}}}{x-y} \frac{\mathrm{e}^{\phi_{+} y}}{1-\mathrm{e}^{\pi y}+\mathrm{i} 0} .
\end{aligned}
$$


This representation is valid for $0<\operatorname{Re} \phi_{+}<\pi$ and arbitrary complex $\phi_{-}$. Let us moreover underline that, contrarily to the original Hänkel determinant formula, the parameter $N$ can be extended here from the set of positive integers to the whole complex plane.

We shall now discuss alternative forms for the representation we have just obtained. First, it should be noted that by shifting the integration contour and relabelling the integration variables in each integral in (3.6) this result can also be put in the form

$$
\tilde{Z}_{N}=\operatorname{det}\left(\mathcal{I}-\zeta \mathcal{W}_{N}\right), \quad \zeta=\mathrm{e}^{\mathrm{i}\left(\phi_{-}-\phi_{+}\right)}
$$

with (to shorten the formulae we shall use more compact notations in terms of polynomials)

$$
\begin{aligned}
\mathcal{W}_{N}(x, y)= & N \frac{P_{N}^{(1 / 2)}\left(x ; \phi_{-}\right) P_{N-1}^{(1 / 2)}\left(y ; \phi_{-}\right)-P_{N-1}^{(1 / 2)}\left(x ; \phi_{-}\right) P_{N}^{(1 / 2)}\left(y ; \phi_{-}\right)}{x-y} \\
& \times \frac{\mathrm{e}^{2 \phi_{+} y}}{1+\mathrm{e}^{2 \pi y}} .
\end{aligned}
$$

We see here that as a matter of fact the kernel is a real-valued function for real $\phi_{ \pm}$(since Meixner-Pollaczek polynomials $P_{n}^{(\lambda)}(x ; \phi)$ are real-valued for real $x$ and $\phi)$.

The parametrization of the weights in the form (1.3) with real $\lambda$ and $\eta$ (hence, real $\left.\phi_{ \pm}\right)$is typical for the so-called disordered phase of the model [8]. Thus, the just obtained representation for the partition function, even if valid for arbitrary choice of vertex weights, can be regarded as "adapted" to the disordered phase. The other two physical regimes are ferroelectric and antiferroelectric and they can be obtained by choosing $\phi_{ \pm}$to be purely imaginary.

To obtain corresponding representations it is sufficient to note that in the case of complex $\phi_{+}$the integrals in (3.6) can be evaluated by closing the integration contours upwards (downwards) in the complex plane of the variables $x_{1}, \ldots, x_{n}$ if $\operatorname{Im} \phi_{+}>0$ (if $\operatorname{Im} \phi_{+}<0$ ). As a result, each integral is given by a sum of residues at simple poles of the function $\omega(x)$ lying in the upper (respectively lower) complex half-plane. Considering the case of $\operatorname{Im} \phi_{+}>0$ (the case of $\operatorname{Im} \phi<0$ leading to an essentially equivalent result) we obtain the following representation for the partition function:

$$
\tilde{Z}_{N}=\operatorname{det}\left(\mathcal{I}-\widetilde{\mathcal{V}}_{N}\right)
$$


where $\widetilde{\mathcal{V}}_{N}$ is the integral operator with discrete kernel

$$
\begin{aligned}
\widetilde{\mathcal{V}}_{N}(x, y)=- & \left\{M_{N}\left(x ; 1, \mathrm{e}^{-2 \tilde{\phi}_{-}}\right) M_{N-1}\left(y ; 1, \mathrm{e}^{-2 \tilde{\phi}_{-}}\right)\right. \\
& \left.-M_{N-1}\left(x ; 1, \mathrm{e}^{-2 \tilde{\phi}_{-}}\right) M_{N}\left(y ; 1, \mathrm{e}^{-2 \tilde{\phi}_{-}}\right)\right\} \frac{N \mathrm{e}^{-2 N \tilde{\phi}_{-}}}{x-y} \mathrm{e}^{-2 \tilde{\phi}_{+} y}
\end{aligned}
$$

whose "integration" variables $x, y$ take nonnegative integer values; for $x=y$ the kernel is to be understood in the sense of the Cristoffel-Darboux identity; in the last formula, the standard notation for Meixner polynomials

$$
M_{n}(x ; \beta, c)={ }_{2} F_{1}\left(\begin{array}{c|c}
-n,-x & 1-\frac{1}{c}
\end{array}\right)
$$

has been used [24]. The "tilded" variables are defined as $\phi_{ \pm}=\mathrm{i} \tilde{\phi}_{ \pm}$. The representation (3.13) is valid for $\operatorname{Re} \tilde{\phi}_{+}>0$ and arbitrary complex $\tilde{\phi}_{-}$. The real values of $\tilde{\phi}_{ \pm}$correspond to ferroelectric $\left(\tilde{\phi}_{ \pm}>0\right)$ and antiferroelectric $\left(\tilde{\phi}_{+}>0\right.$, $\left.\tilde{\phi}_{-}<0\right)$ phases of the model.

It is to be mentioned that representation (3.11) can be also obtained directly by employing the formula

$$
\operatorname{coth} \tilde{\phi}=2 \sum_{x=0}^{\infty} \mathrm{e}^{-2 \tilde{\phi} x}, \quad \operatorname{Re} \tilde{\phi}>0
$$

instead of (2.9) and repeating all considerations of the previous Section. The appearance of Meixner polynomials is then quite obvious since for $|c|<1$ they are subject to the orthogonality condition

$$
\sum_{x=0}^{\infty} M_{j}(x ; \beta, c) M_{k}(x ; \beta, c) \frac{(\beta)_{x}}{x !} c^{x}=\frac{c^{-j}}{1-c} \delta_{j k}
$$

and upon setting $\beta=1$ and $c=\mathrm{e}^{-2 \tilde{\phi}}$ the identification of the proper set of orthogonal polynomials is achieved.

We conclude this Section by considering the so-called rational parametrization of the vertex weights, which corresponds to the case in which $a, b$ and $c$ are restricted by the condition $\mathrm{a} \pm \mathrm{b}=\mathrm{c}$. This regime can be obtained through $\mathrm{a}$ suitable limit from vertex weights (1.3). Namely, depending on the choice of the sign in this restriction, one should just substitute $\lambda, \eta \rightarrow \epsilon \lambda, \epsilon \eta$ (for plus sign) or by $\lambda, \eta \rightarrow \pi / 2-\epsilon \lambda, \pi / 2-\epsilon \eta$ (for minus sign) in (1.3) and take the limit $\epsilon \rightarrow 0$, 
after renormalization of the weights by a factor $1 / \epsilon$. We should of course recover in this limit the corresponding result of Ref. [20].

The Hänkel determinant formula for the partition function of the model with rational weights is simply given by formula (1.4) with the rational functions $\lambda, \eta$ instead of sine functions: $\sin (*) \rightarrow(*)$. The Fredholm determinant representation for the partition function in this case involves Laguerre polynomials; the "rational limit" of the kernel (3.12) can be easily found using

$$
\lim _{\epsilon \rightarrow 0} P_{n}^{(1 / 2)}(x / \epsilon ; \epsilon \phi)=L_{n}(-2 \phi x), \quad \lim _{\epsilon \rightarrow 0} \frac{\mathrm{e}^{\epsilon \phi_{+}(y / \epsilon)}}{1+\mathrm{e}^{\pi(y / \epsilon)}}=\mathrm{e}^{\phi_{+} y} \theta(-y),
$$

where $L_{n}(x)$ is Laguerre polynomial, and $\theta(x)$ is Heaviside step-function. The

same result can be obtained from (3.14), too, with the discrete measure turning into the continuous one in the standard way, when performing the rational limit. Explicitly, the partition function in the rational case is given by the Fredholm determinant of the integral operator on the real positive half-axis, defined by the kernel

$$
\mathcal{V}_{\mathcal{N}}(x, y)=-N \frac{L_{N}(\xi x) L_{N-1}(\xi y)-L_{N-1}(\xi x) L_{N}(\xi y)}{x-y} \mathrm{e}^{-y}
$$

where $\xi=\phi_{-} / \phi_{+}$. The result of Ref. [20] is thus reproduced in the rational limit, which corresponds there to the case $q=1$.

\section{The finite-size determinant representation}

The determinant in (2.26) can be also written as that of some $N \times N$ symmetric matrix whose entries are simply connected with the kernel of the integral operator in the Fredholm determinant representation. One can insert (3.1) in the second line of (3.6) and obtain

$$
\begin{aligned}
& \ln \operatorname{det}_{N}\left(I-A_{-}^{-1} A_{+}\right) \\
& \quad=-\sum_{n=1}^{\infty} \frac{1}{n} \int_{-\infty}^{\infty} \ldots \int_{-\infty}^{\infty} \mathcal{K}_{N}^{-}\left(x_{1}, x_{2}\right) \mathcal{K}_{N}^{-}\left(x_{2}, x_{3}\right) \ldots \mathcal{K}_{N}^{-}\left(x_{n}, x_{1}\right) \prod_{l=1}^{n} \omega^{+}\left(x_{l}\right) \mathrm{d} x_{l} \\
& \quad=-\sum_{n=1}^{\infty} \frac{1}{n} \sum_{k_{1}, \ldots, k_{n}=0}^{N-1} V_{k_{1} k_{2}} V_{k_{2} k_{3}} \ldots V_{k_{n} k_{1}} \\
& \quad=\ln \operatorname{det}_{N}(I-V)
\end{aligned}
$$

where entries of the matrix $V$ are

$$
V_{j k}=\int_{-\infty}^{\infty} p_{j}^{-}(x) p_{k}^{-}(x) \omega^{+}(x) \mathrm{d} x
$$


Clearly, this identity is a consequence of the fact that the matrices $A_{-}^{-1} A_{+}$and $V$ are related by some similarity transformation.

Hence, the partition function admits also the representation

$$
\tilde{Z}_{N}=\operatorname{det}_{N}(I-V)
$$

It turns out that in contrast to entries of matrix $H$, entering the original Hänkel determinant representation (1.4), those of the matrix $V$ can be computed explicitly. It is convenient (in analogy with (3.11) ) to introduce the matrix $W$ by

$$
V=\zeta W, \quad \zeta=\mathrm{e}^{\mathrm{i}\left(\phi_{-}-\phi_{+}\right)}=\mathrm{e}^{-2 \mathrm{i} \eta}
$$

Entries of $W$ are defined by the formula

$$
W_{j k}=2 \sin \phi_{-} \int_{-\infty}^{\infty} P_{j}^{(1 / 2)}\left(x ; \phi_{-}\right) P_{k}^{(1 / 2)}\left(x ; \phi_{-}\right) \frac{\mathrm{e}^{2 x \phi_{+}}}{1+\mathrm{e}^{2 \pi x}} \mathrm{~d} x .
$$

As shown in detail in the Appendix, the integral can be evaluated in closed form. Introducing, for the sake of convenience, the notations

$$
\beta=\frac{\sin \phi_{-}}{\sin \phi_{+}}=\frac{\sin (\lambda-\eta)}{\sin (\lambda+\eta)}, \quad \gamma=\frac{\sin \left(\phi_{+}-\phi_{-}\right)}{\sin \phi_{+}}=\frac{\sin 2 \eta}{\sin (\lambda+\eta)},
$$

(note that $\beta$ and $\gamma$ are precisely the quantities entering the $U_{q}\left(\mathfrak{s l}_{2}\right)$-invariant $R$-matrix, see eqs. (2.23) and (2.24), $\beta=\beta(\nu), \gamma=\gamma(\nu))$ the result reads

$$
\begin{aligned}
W_{j k} & =\gamma^{j+k+1} \sum_{n=0}^{\min (j, k)}\left(\begin{array}{l}
j \\
n
\end{array}\right)\left(\begin{array}{l}
k \\
n
\end{array}\right)\left(\frac{\beta}{\gamma}\right)^{2 n+1} \\
& =\beta \gamma^{j+k}{ }_{2} F_{1}\left(\begin{array}{c}
-j,-k \\
1
\end{array} \frac{\beta^{2}}{\gamma^{2}}\right) .
\end{aligned}
$$

It is worth to remind that the representation (4.3) is for the partition function with the weights (2.22); the original normalization can be achieved by multiplying the RHS of (4.3) by the factor $[\sin (\lambda+\eta)]^{N^{2}} \mathrm{e}^{\mathrm{i}(\lambda-\eta) N}$.

The second expression in (4.7) shows that the entries of matrix $W$ are, in fact, Meixner polynomials, see (3.15). On the other hand, from the first expression in (4.7) follows that matrix $W$ can be written as a product of much simpler matrices. Indeed, introducing $N \times N$ matrices

$$
\begin{aligned}
& \left(J_{+}\right)_{n m}=n \delta_{n-1, m} \\
& \left(J_{0}\right)_{n m}=(n+1 / 2) \delta_{n, m} \\
& \left(J_{-}\right)_{n m}=(n+1) \delta_{n+1, m}
\end{aligned}
$$


and taking into account that

$$
\left(\exp \left\{\gamma J_{+}\right\}\right)_{n m}=\gamma^{n-m}\left(\begin{array}{c}
n \\
m
\end{array}\right), \quad J_{-}=\left(J_{+}\right)^{T}
$$

one obtains

$$
W=\exp \left\{\gamma J_{+}\right\} \beta^{2 J_{0}} \exp \left\{\gamma J_{-}\right\} .
$$

Thus, the matrix $W$ is defined by its Gauss decomposition, i.e., as a product of a lower-triangular, a diagonal, and an upper-triangular matrices, respectively.

Matrix $W$ simplifies considerably in the limit $N \rightarrow \infty$. This is a consequence of the fact that the semi-infinite dimensional matrices $J_{ \pm, 0}$ with entries (4.8) satisfy $\mathfrak{s u}(1,1)$ algebra commutation relations

$$
\left[J_{-}, J_{+}\right]=2 J_{0}, \quad\left[J_{ \pm}, J_{0}\right]=\mp J_{ \pm} .
$$

Standard techniques (widely used for instance in the theory of generalized coherent states [26]) can now be applied to (4.10), with the following result

$$
W=\exp \{2 \eta K\} \quad(N=\infty)
$$

where the semi-infinite dimensional matrix $K$ is

$$
K=\frac{1}{\sin \nu}\left(J_{-}+J_{+}-2 \cos \nu J_{0}\right) .
$$

Here (and below) we use "mixed" set of variables, $\nu=\phi_{-}=\lambda-\eta$ and $\eta$ (indeed the most natural ones for the $U_{q}\left(\mathfrak{s l}_{2}\right)$-invariant $R$-matrix, see Eqs. (2.23) $-(2.25)$ ).

The matrix $K$ is the Jacobi matrix corresponding to the recurrence relation of Meixner-Pollaczek, Laguerre or Meixner polynomials depending whether $|\cos \nu|<1,|\cos \nu|=1$ or $|\cos \nu|>1$, respectively. These polynomials are therefore eigenfunctions of $K$ with eigenvalue $x$, and the spectrum of $K$ is thus given by the support of the measure appearing in the orthogonality condition of the corresponding polynomials, which is $\mathbb{R}, \mathbb{R}_{+}$or $\mathbb{Z}_{+}$, respectively (i.e., it is continuous in the first and second case and discrete in the third one). From this point of view the presence of these same polynomials in the kernel of the integral operator in the Fredholm determinant representations is obvious: the integral operator is just the semi-infinite dimensional matrix $\Pi_{N} \exp \{2 \eta K\}$ re-expressed in the basis of the eigenfunctions of $K$; here the $N$-dimensional projector $\Pi_{N}$ is the matrix with its first $N$ diagonal entries equal to one, and all others entries equal to zero.

Another point which is to be discussed is that formula (4.12) tells us that in case $N=\infty$, matrix $W$ (and hence $V$ ) is just the exponential of some "simple" 
matrix. The analogy which come into mind after looking at formulae (4.3) and (4.12) is with a typical result of calculation of traces of certain class of operators acting over the Fock space of $N$ fermions. Namely, given a matrix $A$, and the corresponding quantum operator $\hat{\boldsymbol{A}}$, bilinear in canonical fermion operators, built from matrix $A$ as follows

$$
\hat{\boldsymbol{A}}=\sum_{n, m=0}^{N-1} \hat{c}_{n+1}^{\dagger} A_{n m} \hat{c}_{m+1}, \quad \hat{c}_{n}^{\dagger} \hat{c}_{m}+\hat{c}_{n} \hat{c}_{m}^{\dagger}=\delta_{n m}
$$

it is well-known [27] that

$$
\operatorname{Tr}[\exp \hat{\boldsymbol{A}}]=\operatorname{det}_{N}(I+\exp A) .
$$

To make a connection with formula (4.3), it is to be mentioned that the minus sign in RHS in (4.15) can be acquired by considering supertraces instead of traces; the supertrace is defined as $\operatorname{Str}[*]:=\operatorname{Tr}\left[(-1)^{\hat{\boldsymbol{N}}} *\right]$, where $\hat{\boldsymbol{N}}$ denotes the fermion number operator: $\hat{\boldsymbol{N}}=\sum_{n=1}^{N} \hat{c}_{n}^{\dagger} \hat{c}_{n}$. However, since the fermion number operator commutes with any operator of the form (4.14), one can consider just traces when dealing with the exponentials of such operators. Taking into account this we can therefore write the following trace formula for the partition function

$$
\tilde{Z}_{N}=\operatorname{Tr}\left[\exp \left\{2 \eta \hat{\boldsymbol{L}}_{N}\right\}\right]
$$

with

$$
\hat{\boldsymbol{L}}_{N}=\hat{\boldsymbol{K}}_{N}+\mathrm{i} \frac{\pi-2 \eta}{2 \eta} \hat{\boldsymbol{N}},
$$

where the operator $\hat{\boldsymbol{K}}_{N}$ is built from the matrix

$$
K_{N}=\frac{1}{2 \eta} \ln W .
$$

Note that in contrast to the entries of matrix $W$ those of matrix $K_{N}$ (and hence of $L_{N}$ ) essentially depend on $N$ as indicated by the notations. Operator $\hat{\boldsymbol{K}}_{N}$, though bilinear in fermions, is essentially non-local, thus making the entries of matrix $K_{N}$ for finite $N$ rather complicated quantities. However, formula (4.12) implies that matrix $K_{N}$ simplifies considerably in the case $N=\infty$, and the corresponding operator $\hat{\boldsymbol{K}}:=\hat{\boldsymbol{K}}_{\infty}$ becomes a local one. Explicitly we obtain

$$
\hat{\boldsymbol{K}}=\sum_{n=1}^{\infty} n \hat{\boldsymbol{H}}_{n, n+1}
$$


where

$$
\hat{\boldsymbol{H}}_{n, n+1}=\frac{1}{\sin \nu}\left\{\hat{c}_{n}^{\dagger} \hat{c}_{n+1}+\hat{c}_{n+1}^{\dagger} \hat{c}_{n}-\cos \nu\left(\hat{c}_{n}^{\dagger} \hat{c}_{n}+\hat{c}_{n+1}^{\dagger} \hat{c}_{n+1}\right)\right\}
$$

We recognize in this expression for operator $\hat{\boldsymbol{K}}$ what is known in the literature as a boost, or ladder, operator. To be precise $\hat{\boldsymbol{K}}$ is the positive half-axis part $\hat{\boldsymbol{K}}=\hat{\boldsymbol{B}}^{(+)}$of the total boost operator $\hat{\boldsymbol{B}}=\hat{\boldsymbol{B}}^{(-)}+\hat{\boldsymbol{B}}^{(+)}$, with $\left[\hat{\boldsymbol{B}}^{(+)}, \hat{\boldsymbol{B}}^{(-)}\right]=0$, see [28]. Operator $\hat{\boldsymbol{B}}$ is the boost operator for the model described by the following Hamiltonian

$$
\hat{\boldsymbol{H}}=\sum_{n} \hat{\boldsymbol{H}}_{n, n+1}=\frac{1}{\sin \nu} \hat{\boldsymbol{H}}_{0}-2 \cot \nu \hat{\boldsymbol{N}}
$$

where $\hat{\boldsymbol{H}}_{0}$ is the hopping term. From this formula it is clear that $\nu$, the spectral parameter in the six-vertex model, is here playing the role of a chemical potential rather than a coupling constant. Nevertheless, all the construction above has led us to a description which is quite analogous to what we have in the corner transfer matrix formalism [8], or to a more general extent, in the vertex operator approach to integrable models [22] and angular quantization method in integrable quantum field theory (see, e.g., [29] an references therein).

As previously explained, in the case of finite $N$ operator $\hat{\boldsymbol{K}}_{N}$ is nonlocal (though free-fermionic). One can however still write the partition function in terms of the product of exponentials of local operators. As a generalization of (4.15) the following is also valid

$$
\operatorname{Tr}\left[\prod_{i} \exp \hat{\boldsymbol{A}}_{i}\right]=\operatorname{det}_{N}\left(I+\prod_{i} \exp A_{i}\right)
$$

where operators $\hat{\boldsymbol{A}}_{i}$ are constructed out of matrices $A_{i}$ through formula (4.14). We can therefore write the following "reconstruction" trace formula for the partition function

$$
\tilde{Z}_{N}=\operatorname{Tr}\left[\left(-\mathrm{e}^{-2 \mathrm{i} \eta}\right)^{\hat{\boldsymbol{N}}} \exp \left\{\gamma \hat{\boldsymbol{J}}_{+}\right\} \beta^{2 \hat{\boldsymbol{J}}_{0}} \exp \left\{\gamma \hat{\boldsymbol{J}}_{-}\right\}\right]
$$

with

$$
\hat{\boldsymbol{J}}_{+}=\sum_{n=1}^{N-1} n c_{n+1}^{\dagger} c_{n}, \quad \hat{\boldsymbol{J}}_{0}=\sum_{n=1}^{N}(n-1 / 2) c_{n}^{\dagger} c_{n}, \quad \hat{\boldsymbol{J}}_{-}=\left(\hat{\boldsymbol{J}}_{+}\right)^{\dagger}
$$

The reconstruction formula (4.23) expresses the partition function of the sixvertex model as the trace of a quantum operator built out of fermions, or, modulo 
Jordan-Wigner transformation, of spin-1/2 operators. Note that, in fact, we have derived these expressions for the partition function starting from the Hänkel determinant representation, previously obtained within the Quantum Inverse Scattering Method. The question which arises from the considerations presented here, is how representation (4.23) in terms of fermions (or spins), which are to be interpreted as effective degrees of freedom, could be extracted directly from the basic definition of the model in terms of vertex configurations. An answer to this question might suggest alternative approaches to the open problem of calculation of correlation functions for the model.

\section{Acknowledgments}

We are grateful to N.M. Bogoliubov, A. Cappelli and V. Tognetti for useful discussions. We acknowledge financial support from MIUR COFIN programme and from INFN (Iniziativa Specifica FI11). This work was been partially done within the European Community network "EUCLID" (HPRN-CT-2002-00325). One of us (A.P.) is supported in part by Russian Foundation for Basic Research, under Grant No. 01-01-01045, and by the programme "Mathematical Methods in Nonlinear Dynamics" of Russian Academy of Sciences.

F.C. thanks Euler International Mathematical Institute and Steklov Institute of Mathematics at St. Petersburg for warm hospitality. A.P. is very grateful to Florence Department of I.N.F.N. and Physics Department of the University of Florence for kind hospitality and support that make this collaboration possible.

\section{Appendix}

The integral defining the entries of matrix $W$ in (4.5) is a particular case of the integral

$$
I_{n m}^{(\lambda)}(\tau, \omega ; \phi)=\frac{1}{2 \pi} \int_{-\infty}^{\infty} P_{n}^{(\lambda)}(x ; \tau) P_{m}^{(\lambda)}(x ; \omega)|\Gamma(\lambda+\mathrm{i} x)|^{2} \mathrm{e}^{(2 \phi-\pi) x} \mathrm{~d} x
$$

where $\phi \in(0, \pi)$ and $\lambda$ is assumed to be real and positive, $\lambda>0$. These restriction are important for convergence of the integral. Our aim here is to prove that for arbitrary $\tau$ and $\omega$ the quantity $I_{n m}^{(\lambda)}(\tau, \omega ; \phi)$ has the following expression

$$
\begin{aligned}
I_{n m}^{(\lambda)}(\tau, \omega ; \phi)= & \frac{\Gamma(2 \lambda+n) \Gamma(2 \lambda+m)}{(2 \sin \phi)^{2 \lambda} \Gamma(2 \lambda) n ! m !}\left[\frac{\sin (\tau-\phi)}{\sin \phi}\right]^{n}\left[\frac{\sin (\omega-\phi)}{\sin \phi}\right]^{m} \\
& \times{ }_{2} F_{1}\left(\begin{array}{c}
-n,-m \\
2 \lambda
\end{array} \mid \frac{\sin \tau \sin \omega}{\sin (\tau-\phi) \sin (\omega-\phi)}\right) .
\end{aligned}
$$


Equality (A.2) is a consequence of the orthogonality condition for the MeixnerPollaczek polynomials and of the identity

$$
P_{n}^{(\lambda)}(x ; \tau)=\sum_{k=0}^{n} \frac{\Gamma(n+2 \lambda)}{\Gamma(k+2 \lambda)(n-k) !} \frac{[\sin (\tau-\phi)]^{n-k}(\sin \tau)^{k}}{(\sin \phi)^{n}} P_{k}^{(\lambda)}(x ; \phi)
$$

which can be viewed as the extension of an analogous formula for Laguerre polynomials (see, e.g., Ref. [30], §10.12, Eqn. (40)). Indeed, using identity (A.3) for both polynomials under the integration sign and employing the orthogonality condition (2.14) one immediately obtains

$$
\begin{aligned}
& I_{n m}^{(\lambda)}(\tau, \omega ; \phi)=\frac{[\sin (\tau-\phi)]^{n}[\sin (\omega-\phi)]^{m}}{2^{2 \lambda}(\sin \phi)^{n+m+2 \lambda}} \\
& \quad \times \sum_{k=0}^{\min (n, m)} \frac{\Gamma(n+2 \lambda) \Gamma(m+2 \lambda)}{\Gamma(k+2 \lambda)(n-k) !(m-k) ! k !}\left[\frac{\sin \tau \sin \omega}{\sin (\tau-\phi) \sin (\omega-\phi)}\right]^{k} .
\end{aligned}
$$

Rewriting the finite sum here as a truncated hypergeometric series results in expression (A.2).

Let us prove now the identity A.3. To simplify as much as possible the combinatorics, let us consider the three-term relation satisfied by the MeixnerPollaczek polynomials:

$$
\begin{aligned}
& (n+1) P_{n+1}^{(\lambda)}(x ; \phi)-[2 x \sin \phi+2(n+\lambda) \cos \phi] P_{n}^{(\lambda)}(x ; \phi) \\
& +(n+2 \lambda-1) P_{n-1}^{(\lambda)}(x ; \phi)=0 .
\end{aligned}
$$

Let us define

$$
S_{n}(x ; \phi)=(\sin \phi)^{-n} P_{n}^{(\lambda)}(x ; \phi)
$$

Recurrence relation (A.5) takes the form

$$
\begin{aligned}
& (n+1) S_{n+1}(x ; \phi)-2(n+\lambda) \cot \phi S_{n}(x ; \phi) \\
& \quad+(n+2 \lambda-1)\left[1+(\cot \phi)^{2}\right] S_{n-1}(x ; \phi)=2 x S_{n}(x ; \phi) .
\end{aligned}
$$

From this relation it is clear that $S_{n}(x ; \phi)$ depends on $\phi$ only through $\cot \phi$, and, moreover, it is a polynomial of order $n$ in $\cot \phi$. It is useful to consider the case $\phi=\pi / 2$ so that $\cot \phi=0$. Denoting $S_{n}(x)=S_{n}(x ; \pi / 2)$, in this case one has

$$
(n+1) S_{n+1}(x)+(n+2 \lambda-1) S_{n-1}(x)=2 x S_{n}(x)
$$


Let us consider the semi-infinite dimensional matrices

$$
\begin{aligned}
& \left(J_{-}\right)_{n m}=(n+1) \delta_{n+1, m} \\
& \left(J_{0}\right)_{n m}=(n+\lambda) \delta_{n, m} \\
& \left(J_{+}\right)_{n m}=(n+2 \lambda-1) \delta_{n-1, m}
\end{aligned}
$$

where, as in the main text of the paper, $n, m \in\{0,1,2, \ldots\}$. These matrices obey $\mathfrak{s u}(1,1)$ algebra commutation relations (4.11). Modulo a diagonal similarity transformation, they correspond to the standard matrix realization of the positive discrete representation $\mathcal{D}^{(+)}(\lambda)$ of $\mathfrak{s u}(1,1)$. In terms of matrices (A.9) relations (A.7) and (A.8) read

$$
\begin{aligned}
& {\left[J_{-}-2 \cot \phi J_{0}+\left(1+(\cot \phi)^{2}\right) J_{+}\right] \vec{S}(x ; \phi)=2 x \vec{S}(x ; \phi)} \\
& {\left[J_{-}+J_{+}\right] \vec{S}(x)=2 x \vec{S}(x),}
\end{aligned}
$$

where $n$-th component of $\vec{S}(x, \phi)$ is just $S_{n}(x, \phi)$, and $\vec{S}(x) \equiv \vec{S}(x, \pi / 2)$. Using the commutation relations of $\mathfrak{s u}(1,1)$ algebra it can be straightforwardly checked that the following relation is valid

$$
\exp \left\{\alpha J_{+}\right\}\left[J_{-}+J_{+}\right]=\left[J_{-}-2 \alpha J_{0}+\left(1+\alpha^{2}\right) J_{+}\right] \exp \left\{\alpha J_{+}\right\}
$$

Thus, by identifying $\alpha=\cot \phi$ we obtain

$$
\vec{S}(x ; \phi)=\exp \left\{\cot \phi J_{+}\right\} \vec{S}(x)
$$

This is a key identity to prove relation (A.3). Indeed relation (A.12) implies that

$$
\vec{S}(x ; \tau)=\exp \left\{(\cot \tau-\cot \phi) J_{+}\right\} \vec{S}(x ; \phi) .
$$

Then, taking into account that

$$
\left(\exp \left\{\alpha J_{+}\right\}\right)_{n m}=\frac{\Gamma(n+2 \lambda)}{\Gamma(m+2 \lambda)(n-m) !} \alpha^{n-m}
$$

one obtains

$$
S_{n}(x ; \tau)=\sum_{m=0}^{n} \frac{\Gamma(n+2 \lambda)}{\Gamma(m+2 \lambda)(n-m) !}(\cot \tau-\cot \phi)^{n-m} S_{m}(x ; \phi) .
$$

Rewriting the last equation in terms of the standard Meixner-Pollaczek polynomials, see (A.6), one arrives finally to identity (A.3), which is thus proved. 


\section{References}

[1] V.E. Korepin, Calculations of norms of Bethe wave functions, Commun. Math. Phys. (1982) 86 391-418.

[2] A.G. Izergin, Partition function of the six-vertex model in the finite volume, Sov. Phys. Dokl. 32 (1987) 878-879.

[3] A.G. Izergin, D.A. Coker and V.E. Korepin, Determinant formula for the six-vertex model, J. Phys. A: Math. Gen. 25 (1992) 4315-4334.

[4] E.H. Lieb, Exact solution of the F model of an antiferroelectric, Phys. Rev. Lett. 18 (1967) 1046-1048.

[5] E.H. Lieb, Exact solution of the two-dimensional Slater KDP model of a ferroelectric, Phys. Rev. Lett. 19 (1967) 108-110.

[6] B. Sutherland, Exact solution of a two-dimensional model for hydrogenbonded crystals, Phys. Rev. Lett. 19 (1967) 103-104.

[7] E.H. Lieb and F.Y. Wu, in Phase Transitions and Critical Phenomena, Vol. 1, edited by C. Domb and M. S. Green, Academic Press, London, 1972, p 321.

[8] R.J. Baxter, Exactly Solved Models in Statistical Mechanics, Academic press, San Diego, 1982.

[9] G. Kuperberg, Another proof of the alternative-sign matrix conjecture, Internat. Math. Res. Notices 1996 (1996) 139-150.

[10] D. Zeilberger, Proof of the refined alternating sign matrix conjecture, New York J. Math 2 (1996) 59-68.

[11] D.M. Bressoud, Proofs and Confirmations: The Story of the Alternating Sign Matrix Conjecture, Cambridge University Press, Cambridge, 1999.

[12] H. Cohn, N. Elkies and J. Propp, Local statistics for random domino tilings of the aztec diamond, Duke Math. J. 85 (1996) 117-166.

[13] W. Jockush, J. Propp and P. Shor, Random domino tilings and the arctic circle theorem, arXiv: math.CO/9801068. 
[14] V.E. Korepin and P. Zinn-Justin, Thermodynamic limit of the six-vertex model with domain wall boundary conditions, J. Phys. A: Math. Gen. 33 (2000) 7053-7066.

[15] P. Zinn-Justin, Six-vertex model with domain wall boundary conditions and one-matrix model, Phys. Rev. E62 (2000) 3411-3418.

[16] N.M. Bogoliubov, A.V. Kitaev and M.B. Zvonarev, Boundary polarization in the six-vertex model, Phys. Rev. E65 (2002) 026126.

[17] N. M. Bogoliubov, A.G. Pronko, and M.B. Zvonarev, Boundary correlation functions of the six-vertex model, J. Phys. A: Math. Gen. 35 (2002) 55255541.

[18] A.R. Its, A.G. Izergin, V.E. Korepin, and N.A. Slavnov, Differential equations for quantum correlation functions, Int. J. Mod. Phys. B4 (1990) 10031037.

[19] V.E. Korepin, N.M. Bogoliubov and A.G. Izergin, Quantum Inverse Scattering Method and Correlation Functions Cambridge University Press, Cambridge, 1993.

[20] N.A. Slavnov, Fredholm determinant representation for the partition function of the six-vertex model, Zap. Nauchn. Sem. POMI 269 (2000) 308-321; arXiv: cond-mat/0005298.

[21] L.D. Faddeev, Algebraic aspects of Bethe-Ansatz, Int. J. Mod. Phys. A10 (1995) 1845-1878.

[22] M. Jimbo, T. Miwa, Algebraic Analysis of Solvable Lattice Models Amer. Math. Soc., Providence RI., 1995.

[23] G. Szegö, Orthogonal Polynomials, Fourth edition, Colloquium Publications, Vol. 23, Amer. Math. Soc., Providence RI., 1975.

[24] R. Koekoek, and R.F. Swarttouw, The Askey-scheme of hyperheometric orthogonal polynomials and its $q$-analoque, Report no. 98-17, Delft University of Technology, 1998.

[25] C.A. Tracy, H. Widom, Fredholm Determinants, Differential Equations and Matrix Models, Commun. Math. Phys. 163 (1994) 33-72. 
[26] A. Perelomov, Generalized Coherent States and Their Applications, Springer, Berlin, 1986.

[27] F.A. Berezin, The Method of Second Quantization, Academic Press, Orlando, 1966.

[28] H.B. Thacker, Continuous space-time symmetries in a lattice field theory, arXiv: hep-lat/9809141.

[29] S. Khoroshkin, A. LeClair, S. Pakuliak, Angular quantization of the SineGordon model at the free fermion point, Adv. Theor. Math. Phys. 3 (1999) 1227.

[30] A. Erdélyi, Higher Transcendental Functions, Vol. II, Robert E. Krieger Publishing Company, Malabar FL, 1981. 